\title{
ARTICLE OPEN Ultrasensitive flexible broadband photodetectors achieving pA scale dark current
}

\author{
Xiao Luo ${ }^{1,2}$, Feiyu Zhao ${ }^{2}$, Lili Du ${ }^{1,2}$, Wenli Lv ${ }^{1}$, Kun $\mathrm{Xu}^{2}$, Yingquan Peng ${ }^{1}$, Ying Wang ${ }^{3}$ and Feiping Lu ${ }^{4}$
}

Organolead halide perovskite is a newly emerging low-cost, solution-processable material with a broadband absorption from the ultraviolet (UV) to visible (Vis) region, which has attracted a great deal of interest in high-performance optoelectronic devices. However, some practicable applications need a cover of UV-Vis-NIR region for photoelectric conversion, a task that remains a significant challenge for further extending the absorption toward the near-infrared radiation (NIR) region. Here, to the best of our knowledge, we prove for the first time an ultrasensitive flexible broadband photodetector based on porous organolead perovskitephthalocyanine heterostructure, which combines the synergetic properties of high UV-Vis absorbance of perovskite with enhanced NIR absorption for triclinic lead phthalocyanine. The photosensitivity of the as-prepared devices reaches up to $10^{4}$ at a low intensity of $10 \mathrm{~mW} \mathrm{~cm}^{-2}$, which is among the largest values reported for broadband photodetectors. Significantly, performed at room temperature, the device achieves a pA scale dark current along with an ultrafast response speed of less than $0.6 \mathrm{~ms}$ for as-adopted full spectra. Our results provide an easy and promising route to develop low-cost, flexible and highly sensitive UV-Vis-NIR photodetectors.

npj Flexible Electronics (2017)1:6; doi:10.1038/s41528-017-0005-x

\section{INTRODUCTION}

Solar energy, mostly spread over visible (Vis) to near-infrared (NIR) region, shows enormous potential in the energy market because of its essentially unlimited supply and renewable, cheap and clean nature. $^{1,2}$ Currently, light detecting and harvesting are two noteworthy photoelectric-conversion processes for exploiting the encouraging solar energy. Here, photodetectors that capture light signals and convert them into electrical signals are important functional units in various energy systems. ${ }^{3-6}$ Among them, broadband photodetectors that can detect light from ultraviolet (UV) to Vis to NIR are critical for color imaging, optical communication, environmental monitoring, and day/night-time surveillance, ${ }^{3,7-10}$ becoming a hot topic recently. However, today commercial inorganic photodetectors have some disadvantages with respect to relatively narrow response spectra and high cost because of the elaborate production process, strongly limiting their broad applications. For instance, silicon photodetectors are mainly used to sense the Vis/NIR light, while silicon carbide-based devices are ordinarily selected for UV sensing. ${ }^{11,12}$ Especially, some IR photodetectors based on narrow-bandgap semiconductors (such as $\operatorname{lnGaAs})^{13}$ must be cooled to utilize due to the abundant intrinsic thermal charge carriers (i.e., larger dark current), complicating the routine use of devices. Therefore, it is urgently desired to obtain a facile and cost-effective UV-Vis-NIR photodetector, achieving room-temperature operational photoelectricconversion.

Fortunately, organolead halide perovskites with a $\mathrm{MAPbX}_{3}(\mathrm{MA}$ $=\mathrm{CH}_{3} \mathrm{NH}_{3}^{+} ; \mathrm{X}=\mathrm{Cl}^{-} / \mathrm{I}^{-} / \mathrm{Br}^{-}$) structure have been proven to be a promising class of materials for optoelectronic devices, including solar cells, ${ }^{14}$ light-emitting diodes, ${ }^{15,}{ }^{16}$ photodetectors, ${ }^{17-19}$ and lasers, ${ }^{20}$ owing to their excellent optical and electrical properties with large absorption cross-section, long photocarrier diffusion length, low trapping density, and high charge mobility. ${ }^{21-24}$ Meanwhile, they also remain effective solution processability and compatibility with flexible substrates. ${ }^{18}$ However, the spotlight perovskites, $\mathrm{MAPbX}_{3}$, can absorb only the photons in the UV-Vis region caused by their wide bandgaps (larger than $1.6 \mathrm{eV})^{24,25}$, restricting some long-wave applications. ${ }^{26}$ The further extension or modulation of the absorption spectrum (in particular toward the NIR) is of interest for the implementation of organohalide perovskites in photoelectric devices. ${ }^{8,} 27,28$ Therefore, an alternative method should be proposed to achieve broader coverage for UV-Vis-NIR region by combining $\mathrm{MAPbX}_{3}$ with other narrow-bandgap materials. Metal phthalocyanine is a class of organic dyes which can be easily obtained to satisfy this condition in practical applications. ${ }^{29}$ Lead phthalocyanine $(\mathrm{PbPc})$ is a shuttlecock-shaped molecule with a high absorption coefficient in the NIR region, which can be further enhanced by means of triclinic absorption induced by selected molecule template or improved substrate temperature. ${ }^{30-32}$ We have successfully demonstrated highly efficient NIR absorption in organic phototransistors $^{32}$ and upconversion devices ${ }^{33}$ based on the proposed strategies above.

Given the above-mentioned advantages of the material's properties, we fabricate and characterize a lateral photoconductor made of heterostructure of organolead perovskite $\left(\mathrm{CH}_{3} \mathrm{NH}_{3} \mathrm{Pbl}_{3-x}\right.$ $\mathrm{Cl}_{x}$ ) and phthalocyanine films, which combine the synergetic properties of high UV-Vis absorbance of perovskite with

\footnotetext{
${ }^{1}$ Institute of Microelectronics, College of Optical and Electronic Technology, China Jiliang University, Xueyuan Street 258\#, Hangzhou 310018, China; ${ }^{2}$ Institute of Microelectronics,

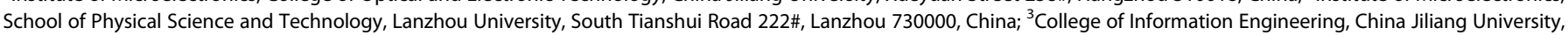
Xueyuan Street 258\#, Hangzhou 310018, China and ${ }^{4}$ Department of Physics, Tianshui Normal University, Tianshui 741001, China

Correspondence: Yingquan Peng (yqpeng@lzu.edu.cn)

Xiao Luo and Feiyu Zhao contributed equally to this work
}

Received: 18 November 2016 Revised: 27 January 2017 Accepted: 27 February 2017

Published online: 16 October 2017 


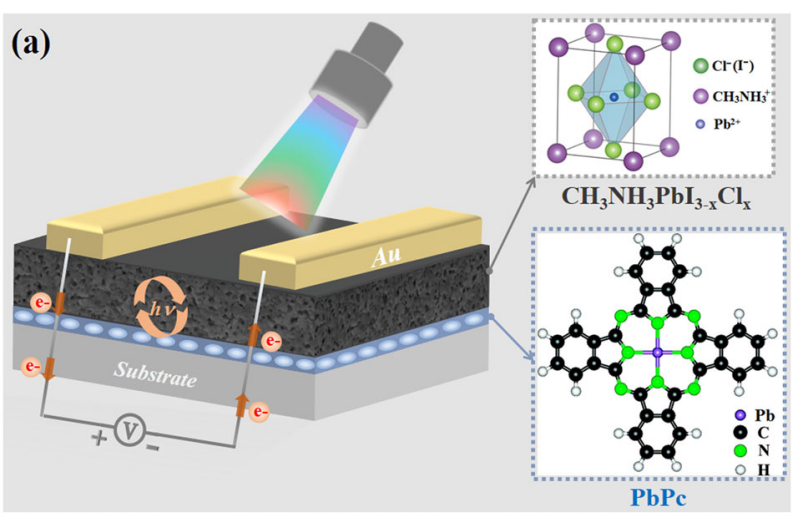

(b)

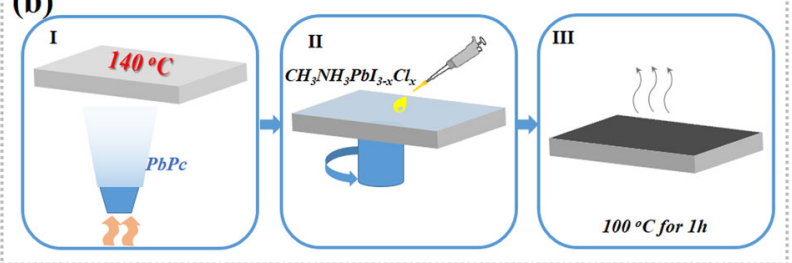

Fig. 1 Schematic configuration and fabrication for achieving an organolead halide perovskite-phthalocyanine heterostructured photodetector. a Schematic diagram of $\mathrm{PbPc} / \mathrm{MAPbl}_{3-x} \mathrm{Cl}_{x}$ photodetector. b Fabrication scheme for the formation of $\mathrm{PbPc} / \mathrm{MAPbl}_{3-x}$ $\mathrm{Cl}_{x}$ films

enhanced NIR light-absorbing for triclinic PbPc. The as-prepared device shows remarkable photosensitivities from the UV to NIR range with ultralow dark current. Note that this ordinary lateral design not only simplifies the use of transparent electrodes as incident window and interface modification materials in vertical sandwich structure, ${ }^{5,26}$ but it is also not restricted by the polarity of applied voltage, which is commonly reverse-biased in verticalstructure photodetectors $3,5,26$, in spite of the fact that the vertical structure can achieve a faster response time owing to its shorter transport path for charge carriers. This methodology, in view of materials and architectural design, provides a reference to achieve facile, cost-effective and flexible broadband photodetectors with superior photosensitive performance.

\section{RESULTS}

Device characterizations

Figure 1a displays the three-dimensional scheme of $\mathrm{PbPc} / \mathrm{MAPbl}_{3}$ ${ }_{-x} \mathrm{Cl}_{x}$ heterostructured photodetector and the corresponding molecular structure of as-adopted materials, respectively. A simple two-step process is adapted here to form $\mathrm{PbPc} / \mathrm{MAPbl}_{3-x} \mathrm{Cl}_{x}$ thin film, as illustrated in Fig. 1b. First, FESEM images are obtained to observe the morphology and microstructure of the heterostructured $\mathrm{PbPc} / \mathrm{MAPbl}_{3-x} \mathrm{Cl}_{x}$ as shown in Figs $2 \mathrm{a}, \mathrm{b}$. The thermally evaporated $\mathrm{PbPc}$ film is identified to have a biggish crystal grain size $(\sim 100 \mathrm{~nm})$ with dense and uniform shape, which indicates that a better crystallinity with less grain boundary is obtained here. ${ }^{32}$ It has been established that the average grain size of organic film increases with substrate temperature and the charge mobility is higher inside grain. ${ }^{32,34,35}$ In contrast, the surface of perovskite films shows micrometer-sized porous structure instead of compact film (Supplementary Fig. S3b versus 3a) to form random networks (Fig. 2b). This is an optimized design for controlling perovskite morphology by using an analogous dilutesolution-phase method. ${ }^{36}$ It has been reasonably revealed that the porous structure of perovskite layer has positive impacts on the device broadband photosensitivity. This is different from those design requirements of solar cells or LEDs based on compact perovskite films. ${ }^{37,} 38$ On one hand, it is useful to achieve a larger surface-to-volume ratio and an easier light penetration from upper perovskite to $\mathrm{PbPc}$ layer, thereby enhancing photo-absorbing of the heterostructured films with limited thickness. On the other hand, physical boundaries among the porous structure can impede carriers' effective transport in darkness. Thus, a relatively low dark current is expected to achieve in the architecture. ${ }^{36} \mathrm{~A}$ control device with compact perovskite films is fabricated simultaneously to verify this analysis. As shown in Supplementary Fig. S4a, the heterostructured device with compact perovskite films shows a $\sim 20$ times higher dark current $(\sim 1 \mathrm{nA})$ than the experimental device with porous perovskite films. Although the control device achieves a relatively improved photocurrent (Supplementary Fig. S4b), the photosensitivity $(P)$ will be far less than that of experimental device (Supplementary Fig. S4c), which is disadvantageous for weak light detection (section 5 of Electronic Supplementary Information, ESI). The result of energy dispersive analysis system of X-ray confirms the element distribution of $\mathrm{CH}_{3} \mathrm{NH}_{3} \mathrm{Pbl}_{3-x} \mathrm{Cl}_{x}$ (Supplementary Fig. $\mathrm{S} 3 \mathrm{C}$ ) and no other foreign elements are presented. $X$ ray diffraction (XRD) patterns of the $\mathrm{PbPc}$ powder and films prepared at different substrate temperatures $\left(T_{\text {sub }}\right)$ are depicted in Fig. $2 \mathrm{C}$, which indicates that $\mathrm{PbPc}$ raw powder and film (deposited at $T_{\text {sub }}=25^{\circ} \mathrm{C}$ ) is mainly monoclinic phase (200); ${ }^{31,39}$ whereas, a triclinic phase is observed by depositing PbPc at $T_{\text {sub }}=140{ }^{\circ} \mathrm{C}$, assigned to triclinic (100). ${ }^{31,32}$ Previous publications have manifested that triclinic $\mathrm{PbPc}$ has a strong absorption band in NIR region, suggesting $\mathrm{PbPc}$ can be used as NIR photosensitizer. ${ }^{31,32,39}$ In addition, XRD patterns of both $\mathrm{MAPbl}_{3-x} \mathrm{Cl}_{x}$ and $\mathrm{PbPc} / \mathrm{MAPbl}_{3-x} \mathrm{Cl}_{x}$ films have similar features, showing two main peaks at $2 \theta=14.18^{\circ}$ and $28.51^{\circ}$ associated with the (110) and (220) planes of perovskite crystals, respectively. ${ }^{14}$ This result reflects that $\mathrm{MAPbl}_{3-x} \mathrm{Cl}_{x}$ film is polycrystalline, and $\mathrm{PbPc}$ molecule has no significant impact on the crystalline structure of subsequently deposited perovskite. The absorption spectra of as-prepared $\mathrm{PbPc}, \mathrm{MAPbl}_{3-x} \mathrm{Cl}_{x}$ and $\mathrm{PbPc} /$ $\mathrm{MAPbl}_{3-x} \mathrm{Cl}_{x}$ films are displayed in Fig. $2 \mathrm{~d}$. It is clearly observed that an enhanced NIR absorption is achieved for PbPc film deposited at $T_{\text {sub }}=140^{\circ} \mathrm{C}$. Generally, PbPc film will achieve B band $\pi \rightarrow \pi^{*}$ and Q band $n \rightarrow \pi^{*}$ transitions as two main absorption bands upon UV and NIR illuminations, respectively (section 4 of ESI), and the peak at $436 \mathrm{~nm}$ is attributed to the characteristic absorption peak of $\mathrm{PbPc}^{32}$ The absorption spectrum of $\mathrm{MAPbl}_{3-x} \mathrm{Cl}_{x}$ is from 250 to $780 \mathrm{~nm}$ since the bandgap energy of $\sim 1.6 \mathrm{eV}$, which is also in good agreement with the reported results. ${ }^{16,17}$ It is noteworthy that there exists complementary spectra for $\mathrm{PbPc}$ and $\mathrm{MAPbl}_{3-x}$ $\mathrm{Cl}_{x}$ photoactive materials, resulting in a broader coverage of UV-Vis-NIR photoabsorption region. As expected, the absorption spectrum of $\mathrm{PbPc} / \mathrm{MAPbl}_{3-x} \mathrm{Cl}_{x}$ film further corroborates the result of broadband absorption above, coming to be promising for the fabrication of a UV-Vis-NIR photodetector.

\section{Optoelectrical properties of as-prepared devices}

The $\mathrm{PbPc} / \mathrm{MAPbl}_{3-x} \mathrm{Cl}_{x}$-based photodetectors are further studied by $I-V$ characteristics in the dark and under the UV-Vis-NIR monochromatic illuminations, measured at room temperature. Figure 3 a shows the typical $I-V$ curve of the photodetector in the dark, manifesting a sub-nA $(\sim 60 \mathrm{pA}$ at $V=50 \mathrm{~V})$ scale dark-current even at the applied voltage $V= \pm 50 \mathrm{~V}$. This result is in good agreement with the morphology and microstructure analysis described above. Furthermore, we select eight specific lasers (wavelength: 405, 450, 532, 655, 780, 808, 830 and $850 \mathrm{~nm}$ ) to characterize the broadband photosensitive performances of asfabricated devices. As it is exposed to light with differentwavelength lasers at various powers, the photocurrents enhance drastically with similar behavior (Fig. 3 and Supplementary Fig. S5), and a microampere scale photocurrent is recorded under illumination of $405 \mathrm{~nm}$ (UV), $532 \mathrm{~nm}$ (Vis) and $780 \mathrm{~nm}$ (NIR) lasers 

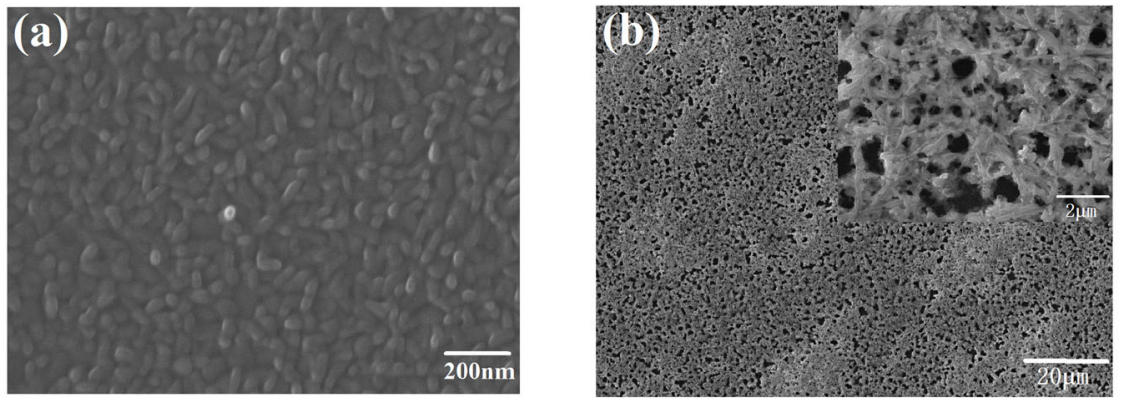

(c)

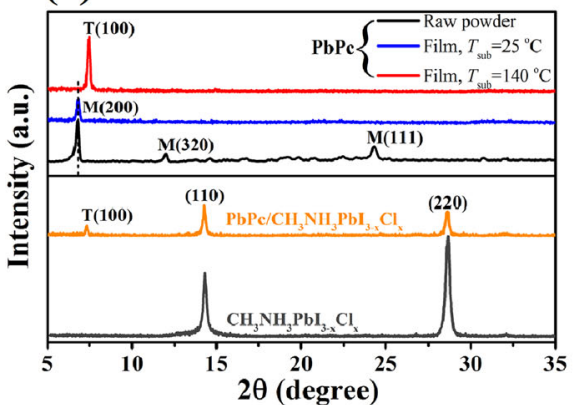

(d)

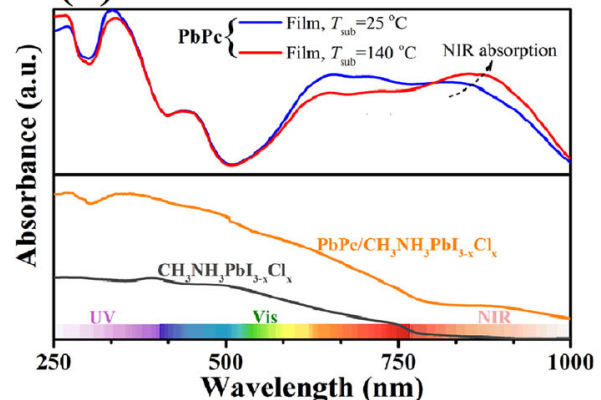

Fig. 2 Properties of organolead perovskite and phthalocyanine. a Representative SEM image of PbPc film evaporated at $140{ }^{\circ} \mathrm{C}$ (substrate temperature). b SEM image of $\mathrm{MAPbl}_{3-x} \mathrm{Cl}_{x}$ perovskite film with porous structure. $\mathbf{c}$ XRD patterns of the PbPc powder and the 50 nm-thick $\mathrm{PbPc}$ films evaporated at 25 and $140{ }^{\circ} \mathrm{C}$ (upper half-plane), $\mathrm{MAPbl}_{3-x} \mathrm{Cl}_{x}$ and $\mathrm{PbPc} / \mathrm{MAPbl}_{3-x} \mathrm{Cl}_{x}$ thin films (lower half-plane). d Absorption spectra of the $50 \mathrm{~nm}$-thick PbPc films evaporated at 25 and $140{ }^{\circ} \mathrm{C}$ (upper half-plane), $\mathrm{MAPbl}_{3-x} \mathrm{Cl}_{x}$ and $\mathrm{PbPc} / \mathrm{MAPbl}_{3}-\mathrm{Cl}_{x}$ thin films (lower half-plane)

at a relatively low power intensity, demonstrating its broadband photodetection characteristics with high sensitivity. It is obvious that the as-prepared photodetectors show significant responsivity for UV-Vis illumination, contributed to an excellent light harvest of $\mathrm{MAPbl}_{3-x} \mathrm{Cl}_{x}$ films in this range ${ }^{5,}{ }^{24}$ meanwhile, a non-negligible photocurrent enhancement in the NIR region is mainly originated from triclinic $\mathrm{PbPc}$ absorption. ${ }^{31,32,39}$ The results above are further corroborated by a detailed performance comparison among single $\mathrm{PbPc}$, single perovskite and $\mathrm{PbPc} /$ Perovskite heterostructured devices, as shown in Supplementary Fig. S6, from which it can be clearly found that the PbPc/Perovskite heterostructure with complementary absorption spectra covering UV-Vis-NIR region will obtain an improved photocurrent and broadband photoresponse. In addition, the thicknesses and deposition sequence of perovskite and $\mathrm{PbPc}$ layer can be important variables for this device due to its photoconductive property and different charge-injection. By changing the thicknesses of perovskite (Supplementary Fig. S7a) or PbPc (Supplementary Fig. S7b), the relative intensity and distribution of photoresponsivity will get changed correspondingly. However, it should be noted that a markedly enhanced dark current will be obtained in the case of $100 \mathrm{~nm}$ PbPc layer due to enhanced bulk conductivity (Supplementary Fig. S7c), which depresses the photosensitivity of the corresponding devices. Likewise, a relatively improved dark current will be achieved when changing the deposition sequence (i.e., Perovskite/PbPc) as a result of an enhanced hole-injection for an ohmic contact (Au/PbPc, Supplementary Fig. S8a). Thus, the device photosensitivity will also be depressed in this framework (Supplementary Fig. S8b).

Another important technological aspect for optoelectronic devices is their time-response stability and speed. The timedependent photoresponse over $120 \mathrm{sec}$ with a 5-period on-off operation is measured under different excitation wavelengths. The heterostructured photodetector shows a stable and repeatable on-off switching in UV-Vis-NIR range (Fig. 4a, c, e and
Supplementary Fig. S9), indicating the excellent photocurrent stability at room temperature. For the application of photodetectors, a fast response and recovery speed is commonly desired character. $^{4,5}$ Herein, the temporal response of our devices is characterized using chopper-generated light pulses (section 7 and Supplementary Fig. S10 in ESI). Figure 4b, d, e show the representative transient photocurrents of this device during the low-frequency $(\sim 150$ and $300 \mathrm{~Hz})$ switching of selected incident light. The dynamic response is noticeable and the rise and fall time are both shorter than $0.6 \mathrm{~ms}$ (Table S1), which can meet lots of application needs. ${ }^{3,4}$ It is noted that the response speed of the $\mathrm{PbPc} / \mathrm{MAPbl}_{3-x} \mathrm{Cl}_{x}$ device is also comparable or even superior to those newly developed broadband photodetectors as listed in Table S2. It should be mentioned that response time of $\sim 0.1 \mathrm{~ms}$ is the present experimental setup limits (mechanical chopper). In order to evaluate the actual response time of as-prepared photodetectors, we used a high-frequency red light-emitting diode $(\sim 300 \mathrm{kHz})$ as excitation source achieved by combining a signal generator (Supplementary Fig. S11a). Thus, an ultrafast response time $(\sim 600 \mathrm{~ns})$ is obtained here (Supplementary Fig. S11b, c), which is comparable or even superior to some recent published results based on perovskite absorbing layer. $5,8,18,19,25,36$ The high repeatability and fast response of the device are promising for large-area photodetector applications.

Time stability and device reproducibility

In order to apply for practical applications, time-stability is a major issue for the as-prepared $\mathrm{PbPc} / \mathrm{MAPbl}_{3-x} \mathrm{Cl}_{x}$ heterostructured photodetector, which is measured by recording the photocurrent changes during storage under ambient conditions for 7 days. The time dependence of $R$ and $P$ for the photodetectors upon illumination with a $655 \mathrm{~nm}$ light at $50 \mathrm{~V}$ is shown in Fig. $5 \mathrm{a}$. The photocurrent almost remains the same as the initial value (Supplementary Fig. S12a). Thus, it can be found that the photoresponsivity almost keeps constant within 7 days, and the 

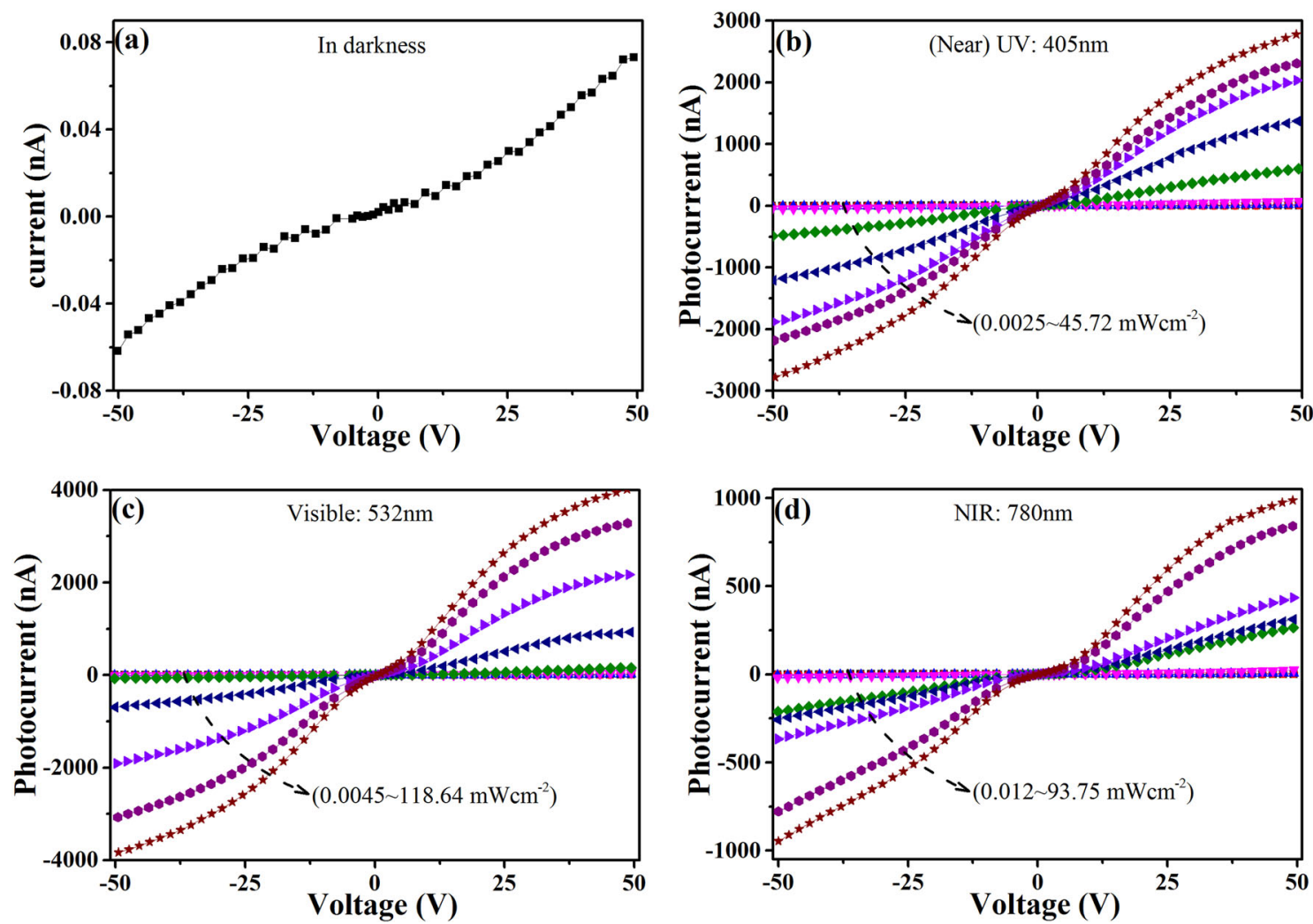

Fig. $3 \quad I-V$ characteristics of perovskite-PbPc photodetectors under dark and different monochromatic light illumination over UV-Vis-NIR range. $\mathbf{a}$ in darkness; $\mathbf{b}$ under Near-UV illumination of $405 \mathrm{~nm}$; c under visual illumination of $532 \mathrm{~nm}$; and d under NIR illumination of $780 \mathrm{~nm}$

fluctuation amplitude is within 1.3\%, which approaches the measurement error. Whereas, the $P$ has a relatively obvious decrease for initial $16 \mathrm{~h}$, and then keeps constant. This behavior possibly results from the moisture and oxygen corrosion, leading to leakage current occurring at those induced defect-sites to enhance dark current (Supplementary Fig. S12a). ${ }^{8}, 17$ Therefore, the timely encapsulation of devices may be essential to enhance the stability of the device for future application. Furthermore, a comparison of environmental stability of single $\mathrm{PbPc}$, single perovskite, and $\mathrm{PbPc}$ / Perovskite heterstructured devices are investigated in detail. One can clearly find that the relative responsivity $(R)$ of the three groups of devices displays a good time stability with a fluctuation of less than $10 \%$ (Supplementary Fig. S12b). In addition, the relative sensitivity variation shows a similar trend to the analysis results above, as shown in Supplementary Fig. S12c. The results above should be attributed to good environmental stability of metal phthalocyanine ${ }^{29}$ and $\mathrm{MAPbl}_{3-x} \mathrm{Cl}_{x}$ mixed halide perovskite (Supplementary Fig. $\mathrm{S} 12 \mathrm{~d})^{40,41}$. Device reproducibility is another key factor for future commercialization. Figure $5 \mathrm{~b}$ gives the distribution of normalized $R$ for as-prepared three groups of devices from two batches of samples. These devices show high reproducibility and uniformity with a $\sim 5 \%$ deviation from the average normalized $R$, and showing $100 \%$ yield.

\section{Physical flexibility}

A flexible photodetector is also fabricated on poly(ethylene terephthalate) (PET) substrate based on the $\mathrm{PbPc} / \mathrm{MAPbl}_{3-x} \mathrm{Cl}_{x}$ heterostructure because the structural frameworks of the organolead perovskite and phthalocyanine are both flexible and deformable. The morphology of $\mathrm{PbPc}$ and perovskite are displayed in Supplementary Fig. S13 after bending manipulation, which indicates that the films remain nearly non-destructive with little cracks. As shown in Fig. 5c, the flexible device clearly illustrates high photosensitivity and the inset further shows a repeatable and stable on-off switching for as-adopted $655 \mathrm{~nm}$ illumination. The device flexibility is studied by bending to a fixed bending angle of about $80^{\circ}$ and repeated bending/recovery manipulation as a bend cycle (Supplementary Fig. S14). After 500 bend cycles, the photodetector still shows high photosensitivity features $\left(\sim 10^{4}\right.$ at $\left.\pm 50 \mathrm{~V}\right)$, as evidenced in Fig. $5 \mathrm{~d}$. Compared to the device in its initial state before bending, the photocurrent and dark current remain nearly unchanged, revealing that the photodetector is not influenced by the external bending stress. Furthermore, the broadband responsivity stability of the flexible photodetector at various bending curvatures is shown in Fig. 5e. The responsivity is almost constant for three different bending states under different light excitation, indicating enormous potential for flexible broadband photodetection. Notably, the flexible device shows no significant decrease in photoresponsivity under continuous bending up to 1000 cycles of different bending curvature radius (Fig. 5f). This simple device structure on flexible substrate suggests its compatibility for scale-up fabrication, which paves the way for commercialization of broadband photodetection technology.

\section{DISCUSSION}

To quantitatively evaluate the performances of as-prepared photodetectors, the photocurrent $\left(I_{\text {ph }}\right)$ and dark current $\left(I_{\text {dark }}\right)$ are first extracted from $I-V$ curves (Fig. 3, Supplementary Fig. S5 and section 6 of ESI), and used to calculate photoresponsivity $(R)$, photosensitivity $(P)$, external quantum efficiency (EQE) and detectivity $\left(D^{*}\right)$. Table S1 summarizes all key metrics for the selected UV-Vis-NIR wavelength illuminations on the $\mathrm{PbPc} /$ $\mathrm{MAPbl}_{3-x} \mathrm{Cl}_{x}$ heterostrctured photodetectors. The as-prepared devices illustrate comparable performance parameters to the 

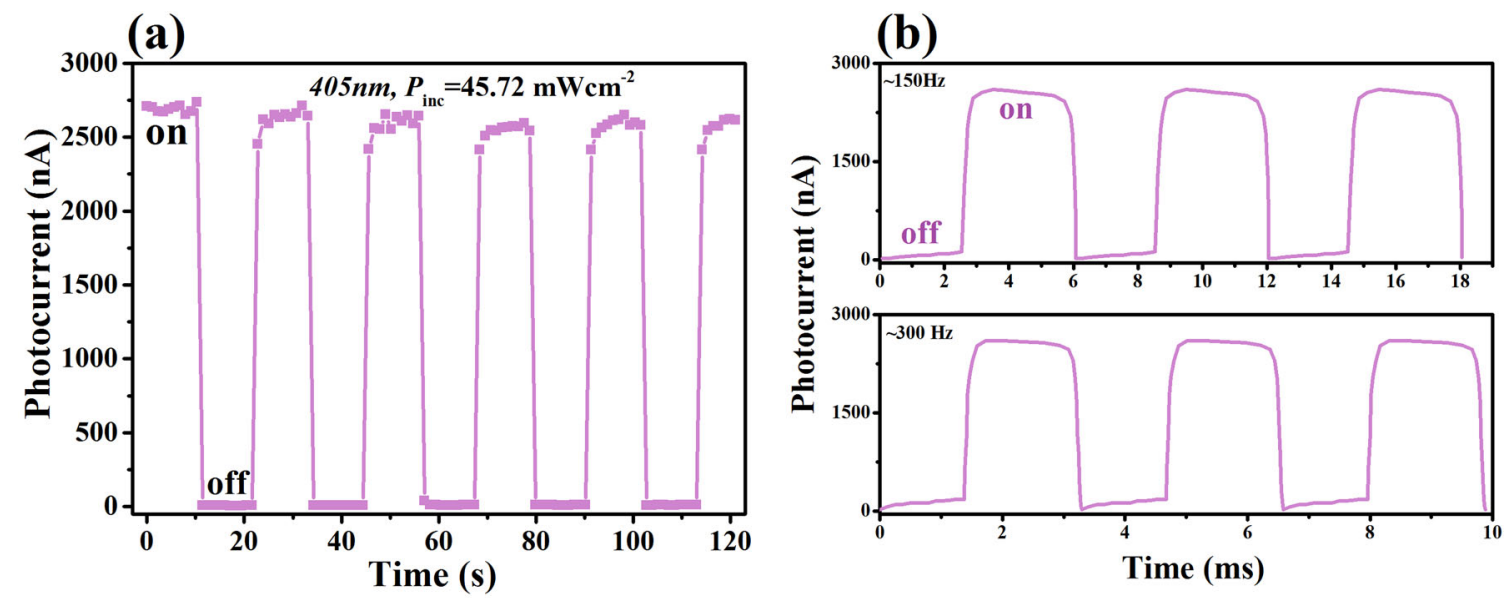

(c)

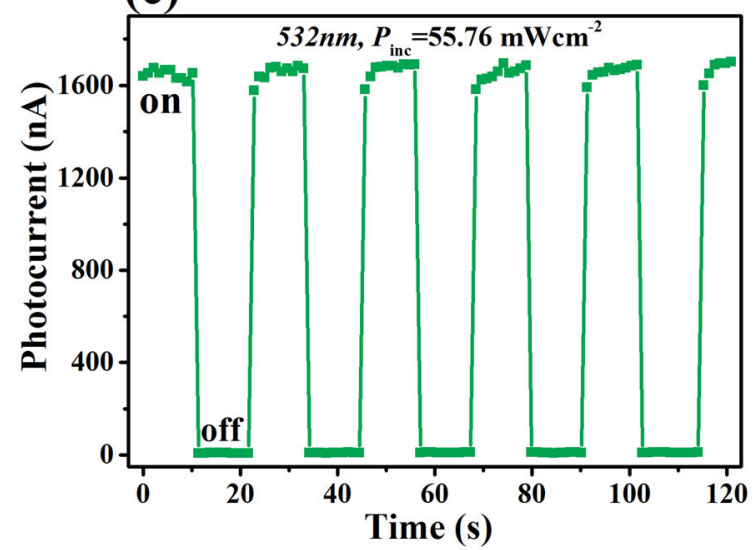

(d)

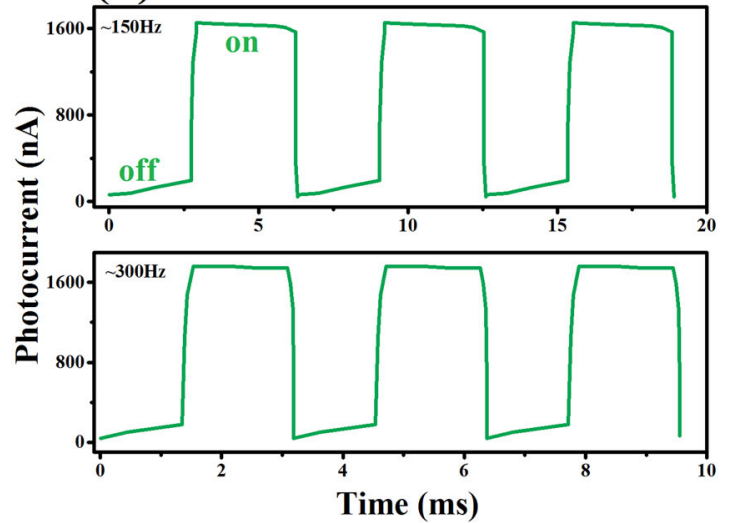

(e)
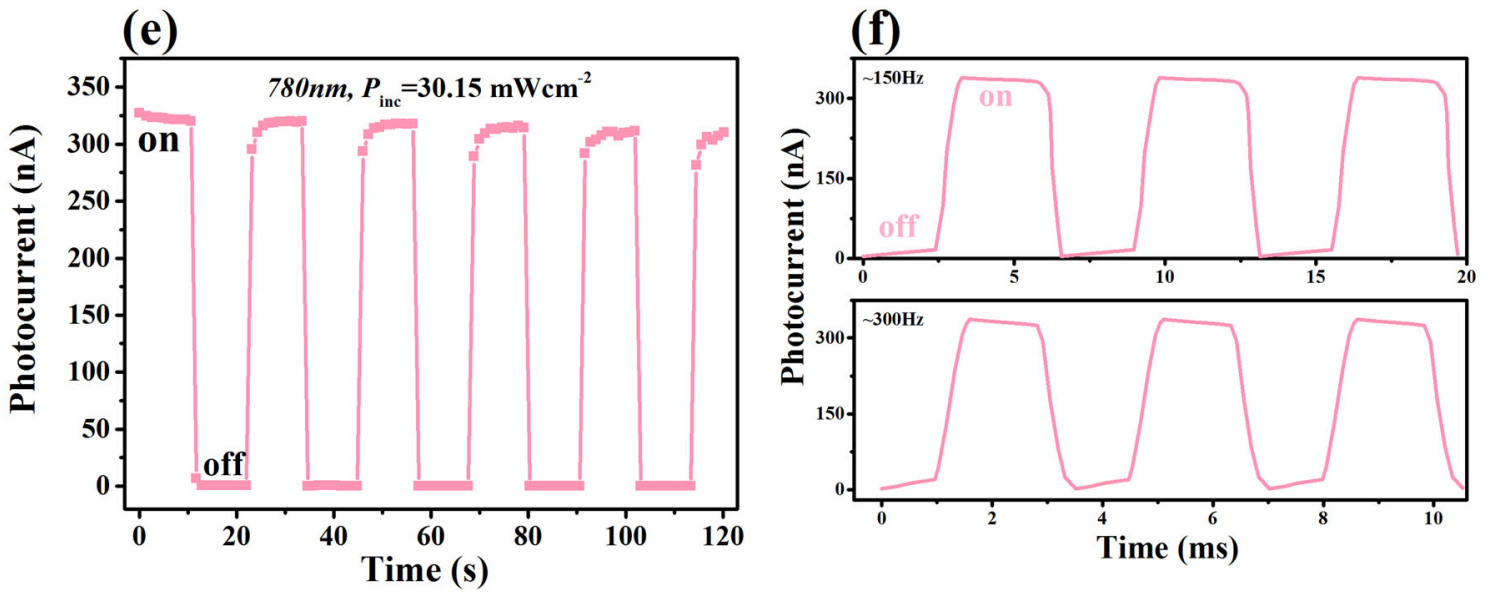

Fig. 4 Time-dependent photocurrent of perovskite-PbPc photodetectors at different wavelengths and photo-switching frequencies with a constant light power and voltage $(V=-50 \mathrm{~V}) . \mathbf{a}, \mathbf{b}, \mathbf{c}, \mathbf{d}$ and $\mathbf{e}, \mathbf{f}$ are corresponding to Near-UV illumination of $405 \mathrm{~nm}$, visual illumination of 532 $\mathrm{nm}$ and NIR illumination of $780 \mathrm{~nm}$, respectively

state-of-the-art broadband detectors (more details in Table S2). $8,9,19,26,30,42,43$ For example, $R$ was calculated to be $554 \mathrm{~mA} \mathrm{~W}^{-1}$ at $405 \mathrm{~nm}$ (UV), $419 \mathrm{~mA} \mathrm{~W}^{-1}$ at $532 \mathrm{~nm}$ (Vis), $130 \mathrm{~mA}$ $\mathrm{W}^{-1}$ at $780 \mathrm{~nm}$ (NIR) at a low power intensity, manifesting its broadband weak-light detection. We further characterize the heterostructured photodetector by varying the excitation power at a fixed excitation wavelength $(450 \mathrm{~nm}$, Fig. 6a). The photocurrent increases superlinearly with increasing incident optical intensity $\left(P_{\text {inc }}\right)$, and it reaches several $\mu \mathrm{A}$ at high excitation powers (30 $\mathrm{mW} \mathrm{cm}^{-2}$ ). Whereas, the $R$ versus $P_{\text {inc }}$ relationship displays a superlinear decrease behavior, which is also commonly observed in some organic and inorganic photodetectors. ${ }^{10,} 17,44$ This effect can be associated with a reduction of the number of photogenerated carriers available for extraction under high light intensity. ${ }^{45}$ Further, the intensity dependent photocurrent and photoresponsivity can be fitted by the power laws $I_{p h} \propto P_{\text {inc }}{ }^{\gamma}$ and $R \propto P_{\text {inc }}{ }^{a-1}, 10,44$ and the $\gamma$ and $a$ are estimated to be 0.80 and 0.79 , which are obviously superior to those values based on previous published semiconductors. ${ }^{10,} 18$ It has been confirmed that the non-unity exponentials are the result of the recombination 

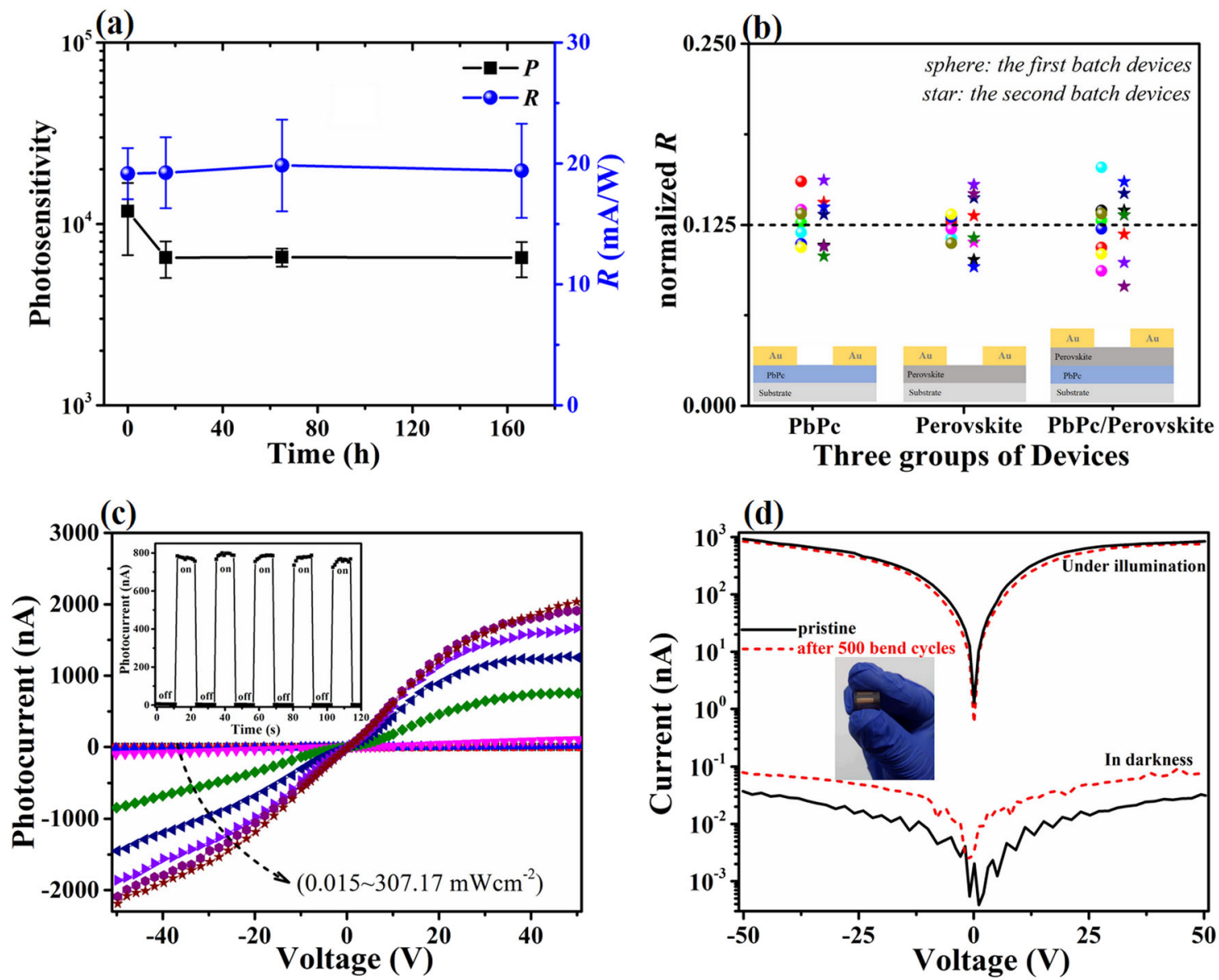

(d)
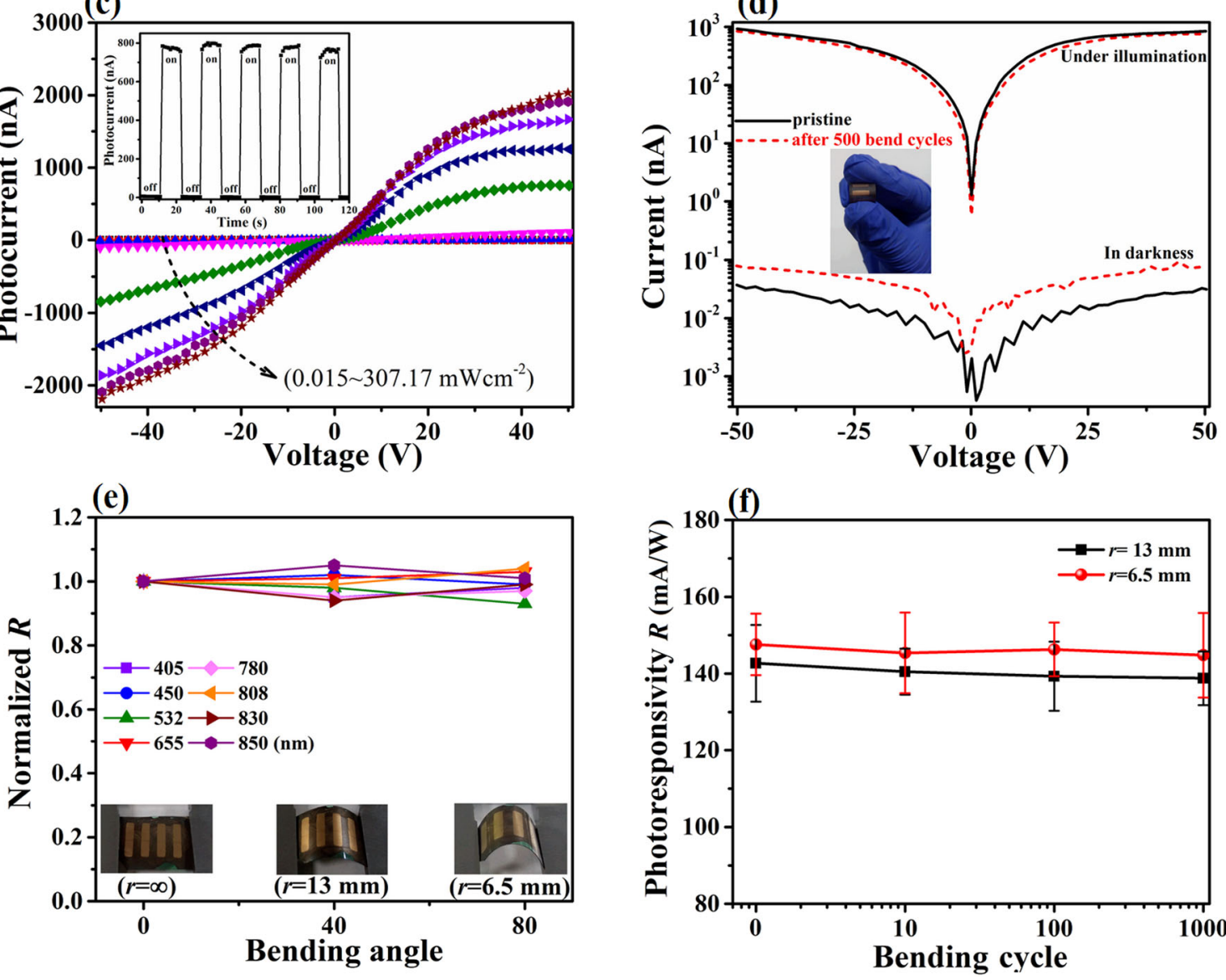

Fig. 5 Device stability, reproducibility, uniformity and flexibility measurements of the perovskite-PbPc series photodetectors. a The photosensitivity and photoresponsivity versus time of exposure in ambient condition for PbPc/Perovskite heterostructured devices. $\mathbf{b}$ The distribution of normalized photoresponsivity $R$ for single PbPc, single perovskite and PbPc/Perovskite heterostructured devices in two batches of samples (a batch of samples have 8 devices). c $I-V$ curves of the flexible device with $\mathrm{PbPc} / \mathrm{MAPbl}_{3-x} \mathrm{Cl}_{\mathrm{x}}$ heterostructure fabricated on transparent PET substrate for $655 \mathrm{~nm}$ illumination; the inset is time-dependent photocurrent measurement at $P_{\text {inc }}=31.66 \mathrm{~mW} \mathrm{~cm}^{-2}$. d $I-V$ curves of the flexible photodetector under dark and illumination without bending and after 500 bend cycles; the inset is the photograph of the flexible photodetector. e Plot of photoresponsivity $R$ versus bending angle (corresponding to curvature radius $r$ ) of the flexible photodetector under broadband light excitation. The insets show corresponding photographs of the device under the different bending states. f Photoresponsivity variations with increasing number of bending cycles at different curvature radius under $655 \mathrm{~nm}$ light excitation

kinetics of photocarriers relating to trap states and coupling effect. ${ }^{6,} 46$ Thus, the higher exponentials mean lower charge recombination or trapping ratios, beneficial to achieve highperformance photodetectors. As expected, it's clearly found that the $\mathrm{PbPc} / \mathrm{MAPbl}_{3-x} \mathrm{Cl}_{x}$-based device is UV-Vis-NIR broad spectral response or detection, even at a low light intensity
$P_{\text {inc }}=0.01 \mathrm{~mW} \mathrm{~cm}^{-2}$ (Supplementary Fig. S15). Based on the pAscale dark current (Fig. 3a), another remarkable feature of this device is the excellent photosensitivity to broadband illuminations. As shown in Fig. $6 \mathrm{~b}$, a minimum $P$ value $(>10)$ can be obtained for full spectrum, significantly up to $10^{4}$ for $450 \mathrm{~nm}$ excitation, which is the one of the maximum values currently 
(a)

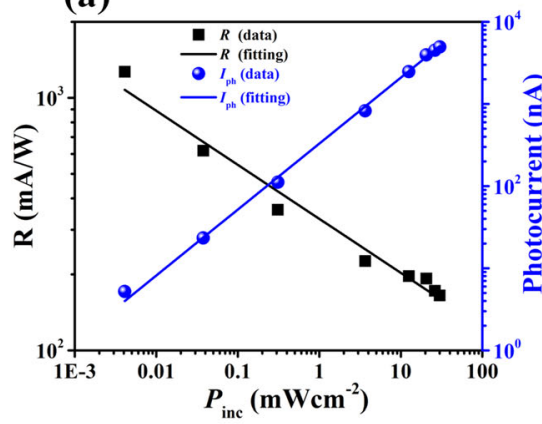

(d)

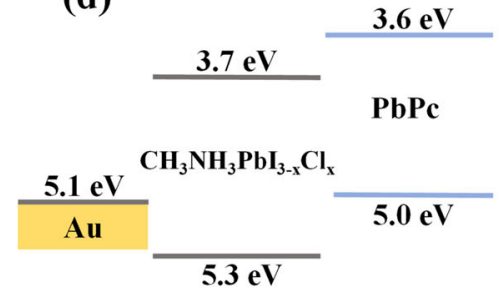

(Schematic energy level) (b)

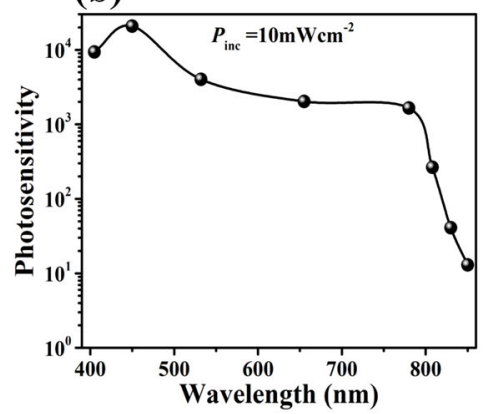

(e)

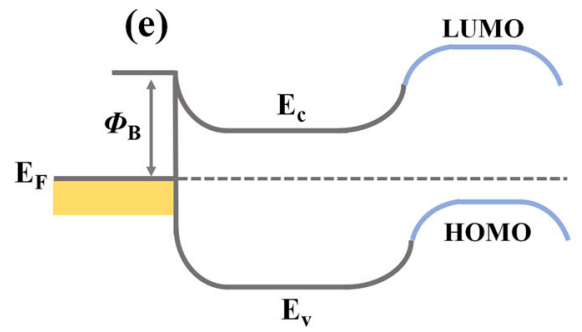

(No illumination equilibrium)

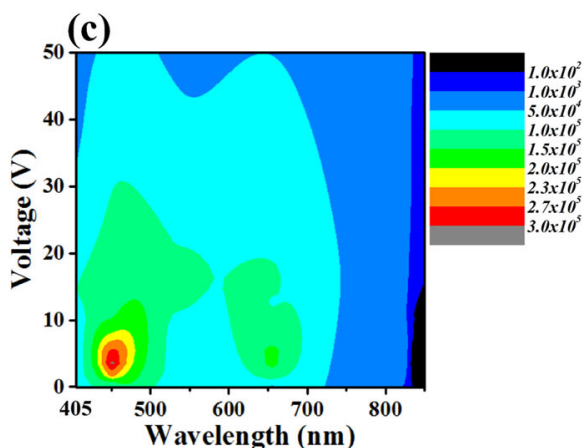

(f)

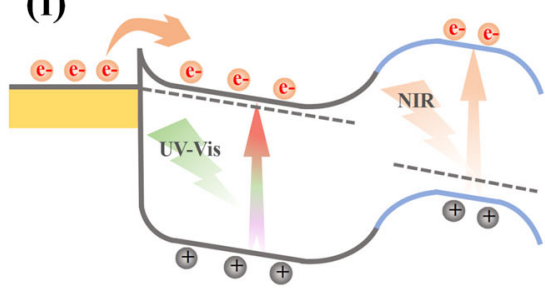

(Under bias and illumination)

Fig. 6 Photosensitive properties and mechanisms of the perovskite-PbPc photodetectors. a Incident power dependence of photoresponsivity and photocurrent under $405 \mathrm{~nm}$ illumination. b The dependence of photosensitivity on the incident wavelength. c Color plot of the maximum photosensitivity of as-prepared device as a function of wavelength and applied bias; the data is extracted from Fig. 3 and Supplementary Fig. S5. d Schematic energy level diagram of the adopted materials; these related values are based on refs. 16,31 . e Band diagram of the $\mathrm{PbPc} / \mathrm{MAPbl}{ }_{3}-\mathrm{Cl}_{x}$ photodetector in darkness. $\mathbf{f}$ Band diagram and charge transport of the $\mathrm{PbPc} / \mathrm{MAPbl}_{3-x} \mathrm{Cl}_{x}$ photodetector under bias and illumination

published. ${ }^{10,36,42,47}$ Furthermore, the color map of $P$ can more clearly and intuitively reveal excellent broadband sensitivity (Fig. 6c). All results above indicate that the PbPc/MAPbl $3-x \mathrm{Cl}_{x^{-}}$ based photodetectors achieve a good photodetection for UV-Vis-NIR region (Table S2). The potential mechanism for the photodetection can be explained by a simplified energy band diagram (Fig. 6d). Firstly, a recent publication has clearly demonstrated that the as-prepared $\mathrm{MAPbl}_{3-x} \mathrm{Cl}_{x}$ perovskite is a strong $\mathrm{N}$-type characteristics of films with a small exciton binding energies of 37-50 meV. ${ }^{22}$ Thus, a P-N heterostructure will form when the perovskite is deposited onto P-type PbPc films, ${ }^{32}$ which may greatly enhance the dissociation of photoexcitons along with lower charge recombination. ${ }^{10}$ The charge transfer via Femi level tuning between the interfaces of $\mathrm{Au} / \mathrm{MAPbl}_{3-x} \mathrm{Cl}_{x}$ and $\mathrm{PbPc} /$ $\mathrm{MAPbl}_{3-x} \mathrm{Cl}_{x}$, resulting in the band bending, and therefore forming Schottky type barrier $\left(\Phi_{\mathrm{B}}\right)$ as well as depletion layers (Fig. 6e), suppressing the dark injection in the heterostructured bilayers. Thus, both the bulk-limited current induced by the porous perovskite/phthalocyanine films and the injection-limited current induced by interfacial barrier lead to ultralow pA-scale dark current, ${ }^{18}$ which is a global device design by considering heterojunction effect and charge-injection interface. Under illumination with the photon energy greater than the energy gap of the semiconductors (Fig. 6f), excitons are generated in $\mathrm{MAPbl}_{3-x} \mathrm{Cl}_{x}$ (UV-Vis) and PbPc (NIR) films, and laterally separated by the applied bias or depletion layers. Electrons are transferred to $\mathrm{N}$-type perovskite, leading to an increase in free carrier density. The increased carrier density in $\mathrm{MAPbl}_{3-x} \mathrm{Cl}_{x}$ film lowers the effective barrier height $\Phi_{B}$ and fills trap states. As a result, bulklimited effect will disappear and injection-limited dominates charge conduction, leading to the easier carriers tunneling and transport than that of devices in darkness, thus, to a significant enhancement in film conductivity and photocurrent. This proposed mechanism has also been successfully used to interpret the performance of other porous-structured photodetectors. ${ }^{9,} 36,48$

In summary, a facile lateral photodetector with bilayer $\mathrm{PbPc} /$ $\mathrm{MAPbl}_{3-x} \mathrm{Cl}_{x}$ heterostructrued films was demonstrated. The as-prepared broadband photodetector displayed ultrahigh roomtemperature photosensitivity with pA-scale dark current in UV-Vis region and extended its photoresponse to the NIR region due to enhanced triclinic absorption of PbPc molecule. More significantly, it also demonstrated moderate environmental stability, reproducibility and favorable mechanical flexibility. We believe that there are no fundamental obstacles to extending the design to other perovskite-organic semiconductors energy conversion systems, which would lead to low-cost, large-area, high-performance broadband photodetectors.

\section{METHODS}

\section{Device fabrication}

The broadband photodetector architecture consisted of the following stack of layers: a transparent substrate, p-type photoactive layer, n-type photoactive layer and lateral gold electrodes. Here, commercial Corning Glass (or flexible PET) was employed as a transparent substrate. $50 \mathrm{~nm} \mathrm{PbPc}$ was thermally evaporated onto the substrates with a substrate-temperature $\left(T_{\text {sub }}\right)$ of $140^{\circ} \mathrm{C}$ in a high vacuum chamber (less than $1.0 \times 10^{-3} \mathrm{~Pa}$ ), on which the organolead halide perovskite $\left(\mathrm{MAPbl}_{3-x} \mathrm{Cl}_{x}\right)$ was prepared by a solution-processed spin-coating method, the detailed fabrication see the Supporting Information. The gold electrodes were thermally evaporated onto the perovskite layer through the shadowed masks.

\section{Device measurements}

$I-V$ measurements were carried out in the dark and illumination condition with commercial UV-Vis-NIR laser diodes. Electrical data were recorded using an organic semiconductor characterization system operated at room temperature, and the intensity of the incident light was monitored by a power meter. More details see the Supporting Information.

\section{ACKNOWLEDGEMENTS}

We would like to thank the anonymous referees whose suggestions and comments largely helped us in improving the original manuscript. We also thank Y-L Liang for assistance in the experiment preparation of high-frequency pulse LED. This work was supported by the National Natural Science Foundation of China Grant No. 10974074, 
61540076, the Research Fund for the Doctoral Program of Higher Education of China Grant No. 20110211110005 and the Research Project of Graduate Teacher of Gansu Province Grant No. 2014B-079.

\section{AUTHOR CONTRIBUTIONS}

X.L. and Y.P. conceived the idea and designed the experiments. X.L. fabricated and characterized the devices, and wrote the paper. F.Z. and L.D. carried out the optical and electrical measurements. F.Z. and K.X. synthesized the perovskite material. W.L., Y.W. and F.L. directed the study. All authors provided valuable feedback.

\section{ADDITIONAL INFORMATION}

Supplementary Information accompanies the paper on the npj Flexible Electronics website (doi:10.1038/s41528-017-0005-x).

Competing interests: The authors declare that they have no competing financial interests.

Publisher's note: Springer Nature remains neutral with regard to jurisdictional claims in published maps and institutional affiliations.

\section{REFERENCES}

1. Wenham, S. R. Applied Photovoltaics. (Routledge, 2012).

2. Cao, W. \& Xue, J. Recent progress in organic photovoltaics: device architecture and optical design. Energy Environ. Sci. 7, 2123-2144 (2014).

3. Haus, J. Optical Sensors. (Wiley Online Library, 2010).

4. Silvano, D. Photodetectors: devices, circuits, and applications. Meas. Sci. Technol. 12, 653 (2001).

5. Dou, L. et al. Solution-processed hybrid perovskite photodetectors with high detectivity. Nat. Commun. 5, 5404 (2014).

6. Zhang, B. Y. et al. Broadband high photoresponse from pure monolayer graphene photodetector. Nat. Commun. 4, 1811 (2013).

7. Saruwatari, M., Grote, N. \& Venghaus, H. Fiber Optic Communication Devices. (Springer, 2001).

8. Chen, S. et al. A Flexible UV-Vis-NIR photodetector based on a perovskite/conjugated-polymer composite. Adv. Mater. 28, 5969-5974 (2016).

9. Yang, J. M., Wang, F. X. \& Pan, G. B. Highly sensitive broadband flexible photodetectors based on a blend film with zinc octaethylporphyrin long nanowires embedded in an insulating polymer. Nanoscale 8, 2811-2818 (2016).

10. Du, L. et al. Toward facile broadband high photoresponse of fullerene based phototransistor from the ultraviolet to the near-infrared region. Carbon 96, 685-694 (2016).

11. Soref, R. A. Silicon-based optoelectronics. Proc. IEEE 81, 1687-1706 (1993).

12. Brown, D. M. et al. Silicon carbide UV photodiodes. IEEE Trans. Electron Devices 40, 325-333 (1993).

13. Yoshizawa, A. \& Tsuchida, H. A $1550 \mathrm{~nm}$ single-photon detector using a thermoelectrically cooled InGaAs avalanche photodiode. Jpn. J. Appl. Phys. 40, 200 (2001).

14. Min, J. et al. Interface engineering of perovskite hybrid solar cells with solutionprocessed perylene-diimide heterojunctions toward high performance. Chem. Mater. 27, 227-234 (2015).

15. Ling, Y. et al. Bright light-emitting diodes based on organometal halide perovskite nanoplatelets. Adv. Mater. 28, 305-311 (2016).

16. Tan, Z. K. et al. Bright light-emitting diodes based on organometal halide perovskite. Nat. Nanotechnol 9, 687-692 (2014).

17. Guo, Y., Liu, C., Tanaka, H. \& Nakamura, E. Air-stable and solution-processable perovskite photodetectors for solar-blind UV and visible light. J. Phys. Chem. Lett. 6, 535-539 (2015)

18. $\mathrm{Hu}, \mathrm{X}$. et al. High-performance flexible broadband photodetector based on organolead halide perovskite. Adv. Funct. Mater. 24, 7373-7380 (2014).

19. Dang, V. Q. et al. Methylammonium lead iodide perovskite-graphene hybrid channels in flexible broadband phototransistors. Carbon 105, 353-361 (2016).

20. Zhu, H. et al. Lead halide perovskite nanowire lasers with low lasing thresholds and high quality factors. Nat. Mater. 14, 636-642 (2015).

21. Wehrenfennig, C., Eperon, G. E., Johnston, M. B., Snaith, H. J. \& Herz, L. M. High charge carrier mobilities and lifetimes in organolead trihalide perovskites. Adv. Mater. 26, 1584-1589 (2014).

22. Lo, M.-F., Guan, Z.-Q., Ng, T.-W., Chan, C.-Y. \& Lee, C.-S. Electronic structures and photoconversion mechanism in perovskite/fullerene heterojunctions. Adv. Funct. Mater. 25, 1213-1218 (2015).

23. Shi, D. et al. Low trap-state density and long carrier diffusion in organolead trihalide perovskite single crystals. Science 347, 519-522 (2015).
24. Brenner, T. M., Egger, D. A., Kronik, L., Hodes, G. \& Cahen, D. Hybrid organicinorganic perovskites: low-cost semiconductors with intriguing charge-transport properties. Nat. Rev. Mater. 1, 15007 (2016).

25. Fang, Y., Dong, Q., Shao, Y., Yuan, Y. \& Huang, J. Highly narrowband perovskite single-crystal photodetectors enabled by surface-charge recombination. Nat. Photonics 9, 679-686 (2015).

26. Qi, J. et al. Panchromatic small molecules for UV-Vis-NIR photodetectors with high detectivity. J. Mater. Chem. C 2, 2431 (2014).

27. Liu, $\mathrm{C}$. et al. Ultrasensitive solution-processed broad-band photodetectors using $\mathrm{CH}_{3} \mathrm{NH}_{3} \mathrm{Pbl}_{3}$ perovskite hybrids and $\mathrm{PbS}$ quantum dots as light harvesters. Nanoscale 7, 16460-16469 (2015).

28. Hao, F., Stoumpos, C. C., Chang, R. P. \& Kanatzidis, M. G. Anomalous band gap behavior in mixed $\mathrm{Sn}$ and $\mathrm{Pb}$ perovskites enables broadening of absorption spectrum in solar cells. J. Am. Chem. Soc. 136, 8094-8099 (2014).

29. Jiang, J. Functional Phthalocyanine Molecular Materials, Vol. 135 (Springer, 2010).

30. Su, Z. et al. High-performance organic small-molecule panchromatic photodetectors. ACS Appl. Mater. Interfaces 7, 2529-2534 (2015).

31. Sakurai, T. et al. Structural control of organic solar cells based on nonplanar metallophthalocyanine/ $\mathrm{C}_{60}$ heterojunctions using organic buffer layers. Org. Electron. 12, 966-973 (2011).

32. Li, Y. et al. Substrate temperature dependent performance of near infrared photoresponsive organic field effect transistors based on lead phthalocyanine. Synth. Met. 205, 190-194 (2015).

33. Lv, W. et al. Organic near-infrared upconversion devices: design principles and operation mechanisms. Org. Electron. 31, 258-265 (2016).

34. Lee, J. Effects of substrate temperature on the device properties of pentacene-based thin film transistors using $\mathrm{Al}_{2} \mathrm{O}_{3+x}$ gate dielectric. J. Appl. Phys. 95, 3733 (2004).

35. $\mathrm{Gu}, \mathrm{W}$. et al. The influences of substrate temperature on ambipolar organic heterojunction transistors. Thin Solid Films 519, 439-442 (2010).

36. Zhang, $\mathrm{Y}$. et al. Ultrasensitive photodetectors based on island-structured $\mathrm{CH}_{3} \mathrm{NH}_{3} \mathrm{Pbl}_{3}$ thin films. ACS Appl. Mater. Interfaces 7, 21634-21638 (2015).

37. $\mathrm{Wu}, \mathrm{Y}$. et al. Perovskite solar cells with $18.21 \%$ efficiency and area over $1 \mathrm{~cm}^{2}$ fabricated by heterojunction engineering. Nat. Energy 1, 16148 (2016).

38. Wang, N. et al. Perovskite light-emitting diodes based on solution-processed selforganized multiple quantum wells. Nat. Photonics 10, 699-704 (2016).

39. Collins, R., Krier, A. \& Abass, A. Optical properties of lead phthalocyanine (PbPc) thin films. Thin Solid Films 229, 113-118 (1993).

40. Antoniadou, M., Siranidi, E., Vaenas, N., Kontos, A. G., Stathatos, E. \& Falaras, P. Photovoltaic Performance and Stability of $\mathrm{CH}_{3} \mathrm{NH}_{3} \mathrm{Pbl}_{3-\mathrm{x}} \mathrm{Cl}_{\mathrm{x}}$ Perovskites. J. Surf. Interfaces Mater. 2, 323-327 (2014).

41. Heo, J. H. \& Im, S. H. Highly reproducible, efficient hysteresis-less $\mathrm{CH}_{3} \mathrm{NH}_{3} \mathrm{Pbl}_{3-\mathrm{x}} \mathrm{Cl}_{x}$ planar hybrid solar cells without requiring heat-treatment. Nanoscale 8 , 2554-2560 (2016).

42. Yoo, J., Jeong, S., Kim, S. \& Je, J. H. A stretchable nanowire UV-Vis-NIR photodetector with high performance. Adv. Mater. 27, 1712-1717 (2015).

43. $\mathrm{Wu}$, J.-J., Tao, Y.-R., Wu, Y. \& Wu, X.-C. Ultrathin $\mathrm{SnS}_{2}$ nanosheets of ultrasonic synthesis and their photoresponses from ultraviolet to near-infrared. Sens. Actuators B Chem. 231, 211-217 (2016).

44. Buscema, M. et al. Fast and broadband photoresponse of few-layer black phosphorus field-effect transistors. Nano Lett. 14, 3347-3352 (2014).

45. Bube, R. H. Photoelectronic Properties of Semiconductors. (Cambridge University Press, 1992).

46. Rose, A. Concepts in Photoconductivity and Allied Problems. (1978).

47. Song, J. et al. Monolayer and few-layer all-inorganic perovskites as a new family of two-dimensional semiconductors for printable optoelectronic devices. Adv. Mater. 28, 4861-4869 (2016).

48. Yan, C., Singh, N. \& Lee, P. S. Wide-bandgap $\mathrm{Zn}_{2} \mathrm{GeO}_{4}$ nanowire networks as efficient ultraviolet photodetectors with fast response and recovery time. Appl. Phys. Lett. 96, 053108 (2010).

Open Access This article is licensed under a Creative Commons Attribution 4.0 International License, which permits use, sharing, adaptation, distribution and reproduction in any medium or format, as long as you give appropriate credit to the original author(s) and the source, provide a link to the Creative Commons license, and indicate if changes were made. The images or other third party material in this article are included in the article's Creative Commons license, unless indicated otherwise in a credit line to the material. If material is not included in the article's Creative Commons license and your intended use is not permitted by statutory regulation or exceeds the permitted use, you will need to obtain permission directly from the copyright holder. To view a copy of this license, visit http://creativecommons. org/licenses/by/4.0/

(c) The Author(s) 2017 